tumour size, larger cancers are compared with smaller ones and screen detected cancers may appear less aggressive simply because of their shorter biological age. However, our results indicate that screen detected breast carcinomas are significantly associated with several features associated with low malignant potential even after adjustment for their smaller size.

Our finding of low malignant potential in screen detected breast cancer is supported by two smaller studies. In one study 37 breast cancers detected by mammography were found to have smaller $S$ phase fractions and less DNA aneuploidy than 60 control cancers diagnosed a few years earlier, but no adjustment for size of the cancers was made. ${ }^{15}$ Similarly, in another study 42 breast cancers found in a palpation based screening were found to have less aggressive histological and cytometric features than in clinical controls. ${ }^{16}$

The lower malignant potential of screen detected breast cancer may decrease the efficiency of screening in saving lives and may at least partially explain why significant reductions in mortality have not always been achieved by screening. ${ }^{4-6}$ If treatment of breast cancer is based only on the size of the primary tumour many women with cancer detected by screening may be overtreated.

This study was supported by the Finnish Foundation for Cancer Research.

I Shapiro S, Venet W, Strax P, Venet L, Roeser R. Selection, follow-up, and analysis in the health insurance plan study: A randomized trial with breast cancer screening. National Cancer Institute Monographs 1985;67:65-74
2 Verbeek ALM, Holland R, Sturmans F, Hendriks JHCL, Mravunac M, Day NE. Reduction of breast cancer mortality through mass screening with modern mammography. First results of the Nijmegen project, 1975-1981. Lancet 1984;ii:1222-4.

3 Tabar L, Fagerberg CJG, Gad A, Baldetorp L, Holmberg LH, Gröntoft O. Reduction in mortality from breast cancer after mass screening with mammography. Lancet 1985; ;:829-32.

4 Andersson I, Aspergren K, Janzon L, Landberg T, Lindholm K, Linell F Mammographic screening and mortality from breast cancer: the Malmö mammographic screening trial. $B M \mathcal{J}$ 1988;297:943-8.

5 Chamberlain J, Coleman D, Ellman R, Moss SM. First results on mortality reduction in the UK trial of early detection of breast cancer. Lancet 1988;ii:411-6.

6 Roberts MM, Aleksander FE, Anderson TJ, Chetty U, Donnan PT, Forrest $P$. Edinburgh trial of screening for breast cancer: mortality at seven years. Lancet 1990;335:241-6.

7 Nielsen M, Thomsen JL, Primdahl S, Dyreborg U, Andersson JA. Breast cancer and atypia among young and middle-aged women: a study of 110 medicolegal autopsies. Brf Cancer 1987;56:814-9.

8 Union of International Cancer Classification. TNM classification of malignan tumours. 4th ed. Geneva: UICC, 1987.

9 World Health Organisation. Histological typing of breast tumours. International classification of tumours No 2. 2nd ed Geneva: WHO, 1981.

10 Toikkanen $S$, Jeensu $H$. Prognotic factors and long-term survival in breast oikkanen S, Joensuu H. Prognostic factors and long-term surviva

11 Hedley DW, Frined urban population. AP, RIS I for analysis of cellular DNA content of paraffin-embedded pathological material using flow cytometry. I Histochem Cytochem 1983;31:1333-5.

12 Camplejohn RS, Macartney JC, Morris RW. Measurement of S-phase Camplejohn RS, Macartney JC, Morris RW. Measurement of S-phase
fractions in lymphoid tissue comparing fresh versus paraffin-embedded tissue and $4^{\prime}, 6^{\prime}$-diamino-2 phenylindole dihydrochloride versus propidium iodide staining. Cytometry 1989;10:410-6.

3 Agresti A. Categorical data analysis. New York: Wiley, 1990.

14 Koscielny S, Tubiana M, Le MG, Valleron AJ, Mouriesse H, Contesso G, et al. Breast cancer: relationship between the size of the primary tumour and probability of metastatic dissemination. Br f Cancer 1984;49:709-15.

15 Kallioniemi OP, Kärkkäinen A, Auvinen O, Mattila J, Koivula T, Hakama M. DNA flow cytiometric analysis indicates that many breast cancers detected in the first round of mammographic screening have a low malignan potential. Int $\mathcal{A}$ Cancer 1988:42:697-702.

16 Joensuu H, Toikkanen S, Klemi PJ. Histological features, DNA content and prognosis of breast carcinoma found incidentally or in screening. Br f Cancer 1991;64:588-92.

(Accepted 27 November 1991 )

\title{
Community surveillance of complications after hernia surgery
}

\author{
Ian S Bailey, Susan E Karran, Kim Toyn, Patricia Brough, Charles Ranaboldo, Stephen J Karran
}

\section{Abstract}

Objective-To assess the effect of a programme of postoperative community surveillance on the rate of detection of wound complications after operation for inguinal hernia.

Design-Prospective audit of wound complications including complications recorded in case notes and those discovered by community surveillance.

Setting-Academic surgical unit of three consultant surgeons.

Patients -510 patients undergoing elective inguinal hernia repair between June 1985 and August 1989.

Results - The wound infection rate recorded in the hospital notes was $3 \%$ compared with $9 \%$ when additional information was obtained from community surveillance. Wound complications were detected in $143(28 \%)$ patients by community surveillance compared with a complication rate of $7 \%$ in the case records for the same patients.

Conclusions - Wound complications are common after clean surgery in patients discharged home early. Complication rates are a reflection not only of the standards of surgical practice but also the rigour with which they are sought. Before national comparative audit data are published the method of collection must be standardised. For short stay surgery this should include meaningful community surveillance.

\section{Introduction}

All surgeons should now be auditing their practice, as dictated by the government and supported by the royal colleges.' The audit process is still evolving and although many publications have suggested various audit methods, no clear consensus has emerged.

Outcome measurements, apart from death, are often difficult to define in a simple, objective manner. Studies assessing treatment in patients who died in the perioperative period, ${ }^{2}$ or after trauma ${ }^{3}$ have identified important problems of quality of care. These studies, however, encompass only a small percentage of surgical patients.

At a local level audit usually comprises crude case load analysis with some assessment of mortality and morbidity. This may be based on data recorded by junior medical staff in an audit book or on structured audit databases developed recently. Such a process may provide adequate performance assessment for many surgical procedures, but assessment is usually less than adequate for the large and increasing number of short stay and day case procedures. Many patients after such surgery are not followed up at all or are seen some four to six weeks later with a "quick chat" and a cursory examination. Problems mentioned postoperatively have usually resolved by the time of the follow up appointment, and the patient is subsequently discharged with no record being made of such events. The development of purchaser-provider arrangements will, however, increasingly dictate that even this quality check may not happen.

It has many times been suggested that wound infection rates after "clean" surgery provide a good indicator of surgical performance, ${ }^{4}$ and more recently it has been suggested that wound infection rates should be published to identify "good" units. The reported incidence of wound infections, however, may well be as much a reflection of the rigour with which patients 
are reviewed as of the excellence of the surgical team

The aim of this study, therefore, was to assess the effect of a prospective audit that included community surveillance on the detection of wound complications after hernia surgery.

\section{Patients and methods}

A prospective study of all elective inguinal hernia repairs performed between June 1985 and August 1989 was undertaken on one surgical unit. Patients were managed in a standard manner on this unit: they were admitted on the day before surgery or the day of surgery and usually discharged home within 48 hours of the operation. For this study, before discharge the wound was graded by an experienced research nurse using a specially devised wound assessment chart (table I). The patient was then reviewed in the community 10-14 days after operation by the research nurse; the wound was regraded and any treatment instituted in the community was recorded. Patients were subsequently reviewed at four to six weeks either in the outpatients department or again in the community. Any complications recorded in the case notes were recorded on the trial form at the late follow up.

Data obtained from this study were subsequently evaluated by an independent observer (ISB) who had not been involved in either the initial care or the follow up of the patients.

The wound grading system used was simplified for the analysis. By using the worst wound score recorded and information about any treatment instituted either in hospital or the community, wounds were regraded in four categories: (a) normal healing; (b) minor complication; (c) wound infection - wounds graded IV or V or wounds treated with antibiotics after discharge from hospital, irrespective of the wound grading given to them by the nurse; and $(d)$ major haematoma-wound or scrotal haematomas requiring aspiration or evacuation.

\section{Results}

Between June 1985 and August 1989, 560 patients were recorded as having undergone elective repair of inguinal hernia. In 28 patients no follow up record form was available; 15 patients were not seen after operation, and a further seven patients were found to have had an epigastric or umbilical hernia repaired rather than an inguinal hernia. Therefore, 510 patients were suitable for this review.

A total of $143(28 \%)$ patients had a local complication recorded during the follow up assessment. Sixty seven $(13 \%)$ were treated for a wound infection. Of these 67, $44(66 \%)$ had a wound graded as grade III (30 patients)

TABLE I-Grading of wounds

\begin{tabular}{ll}
\hline Grade & Appearance \\
\hline 0 & Normal healing \\
I Normal healing with mild brusing or erythema: & Some bruising \\
a & Considerable bruising \\
b & Mild erythema \\
c & At one point \\
II Erythema plus other signs of inflammation: & Around sutures \\
a & Along wound \\
b & Around wound \\
c & At one point only $(\leqslant 2 \mathrm{~cm})$ \\
d & Along wound $(>2 \mathrm{~cm})$ \\
III Clear or haemoserous discharge: & Large volume \\
a & Prolonged $(>3$ days $)$ \\
b & \\
c & \\
d & Major complication \\
IV Pus: & At one point only $(\leqslant 2 \mathrm{~cm})$ \\
a & Along wound $(>2 \mathrm{~cm})$ \\
b & \\
V Deep or severe wound infection with or & without tissue breakdown; \\
haematoma requiring aspiration & \\
\hline
\end{tabular}

TABLE II-Complications of elective hermia repair detected in hospital notes and community follow up. Figures are numbers (percentages)

\begin{tabular}{lcc}
\hline Complications & $\begin{array}{c}\text { In hospital notes } \\
(\mathbf{n}=510)\end{array}$ & $\begin{array}{c}\text { At community follow up } \\
(\mathbf{n}=510)\end{array}$ \\
\hline None & $473(92)$ & $367(72)$ \\
Minort & $15(3)$ & $87(17)$ \\
Wound infection $\ddagger$ & $16(3)$ & $44(9)$ \\
Haematoma & $6(1)$ & $12(3)$ \\
\hline
\end{tabular}

tIncludes wounds without infection treated by general practitioner with antibiotics.

¥Includes only wound infections confirmed by community review.

or IV (14 patients). A further 23 patients (5\%) were treated with antibiotics by their general practitioner for a possible wound infection. Twelve of these patients, however, had had a normal wound score when seen in the community, and the rest had had only a mild bruising or erythema. Sixty patients $(12 \%)$ had wound or scrotal haematomas noted after operation. Most of these (48) were recorded as marked bruising more than 10 days after operation and required no treatment, but 12 patients required operative drainage or aspiration, and 17 complained of a haemoserous discharge postoperatively for which no action was taken and from which no organsims were grown.

Most of the wound complications (105, 73\%) detected during this study were not recorded in the hospital records. The hospital's rate of recording the confirmed wound infections (grades IV and V) and haematomas requiring treatment was only $43 \%(24 / 56)$. This comprised seven (23\%) of the 30 grade IV and nine $(75 \%)$ of the 12 grade $V$ wound infections and $\operatorname{six}(50 \%)$ of the 12 haematomas requiring treatment. Of the minor complications and normal wounds treated with antibiotics, only $15(26 \%)$ of the 87 found in the community had been recorded in the hospital records (table II)

In all, $95 \%(137 / 143)$ of wound complications were apparent at the first community review; $5 \%(7 / 143)$ were detected at the late follow up. Overall, $85 \%$ $(122 / 143)$ of complications had resolved at the six week follow up.

\section{Discussion}

Wound complications are common after inguinal hernia surgery when careful community surveillance, rather than reliance on standard methods of hospital recordkeeping, is used to audit the results of surgery.

Defining a complication is easy at the extremes but often becomes subjective for minor problems. The wound grading form devised for this study allowed a descriptive classification of the different wounds and was found easy to use by the reviewing nurses. The grouping of different grades into the smaller number of categories used in this paper allowed us to describe well recognised wound complications. Many of the complications recorded here may not affect the long term outcome after surgery, and indeed the majority were not detected or recorded by standard hospital follow up. We believe, however, that each of the recorded complications represents suboptimal postoperative recovery and, in many cases, delayed the patient's return to full activity. Similarly, many if not all of these complications used community health care resources, requiring visits to and by general practitioners and district nurses.

Quality of data collection is fundamental to the success of the audit process. This study shows that community surveillance after hernia surgery found a higher than expected incidence of wound complications and that many of these complications were not recorded by the standard follow up procedures. Even though this was a prospective study we still did not have adequate follow up on $10 \%$ of suitable cases, further 
illustrating the difficulty of complete and accurate data collection. ${ }^{2}$

If national publication of institutional wound infection rates is a real possibility a standardised method of audit will clearly be necessary. We have shown that many wound complications after hernia surgery are not recorded in the hospital records and thus will not be audited by case note review. Many complications become apparent after discharge from hospital, are treated in the community, and have resolved by six weeks after operation. How can these problems best be detected?

A hospital based community surveillance team would be expensive to run and may not be necessary. Review of these patients in outpatient departments 10-14 days after operation with specific attention to recording visible and reported complications would have detected some $95 \%$ of the complications. However, it seems that many fundholding general practitioners will negotiate this sort of minor surgery without hospital follow up. Although general practitioners could provide the requisite audit information, we found a high incidence of general practitioners treating hernia wounds with antibiotics when there was little evidence of infection. Clearly, before we advocate publication of audit data, with all its implications, we must investigate more intensively the different methods of data collection.

Previous study of wound infections after hernia surgery report infection rates from $7 \cdot 3 \%$ (reported by the Public Health Laboratory Service at a time when many patients spent $10-14$ days in hospital) 5 to $1 \cdot 3 \%$ (reported in the United States). ${ }^{6}$ The low wound infection rate in the American study is likely to be a reflection of the definition of wound infection, which included wound breakdown but excluded lesser degrees of purulent discharge particularly associated with sutures or minor segments of the wound. We would, however, do well to emulate the results of this study.

In conclusion, this study has shown a high wound complication rate after hernia surgery. The community surveillance programme increased the detection of these complications fourfold. If we are going to audit our results seriously it is clear that new follow up practices are needed for short stay surgery. The optimal method of achieving this will need to be investigated in future research. The method of collection of audit data and the definition of complications must be established at a national level before decisions to publish comparative audit data are made.

We thank our surgical and general practitioner colleagues for their help and advice, in particular Professor Irving Taylor, Mr Gavin Royle, and Mr Colin Johnson, and also Mr Roger Farrow for his support.

1 Secretaries of State for Health, Wales, Northern Ireland, and Scotland. Working for patients. London: HMSO, 1989.

2 Buck N, Devlin HB, Lunn JN. The report of a confidential enquiry into perioperative deaths. London: Nuffield Provincial Hospital Trust and the perioperative deaths.

3 Anderson ID, Woodford M, de Dombal FT, Irving M. Retrospective study of 1000 deaths from injury in England and Wales. BMf 1988;296:1305-8.

4 Cruse P. Surgical infection surveillance. In: Karran SJ, ed. Controversies in surgical sepsis. New York: Praeger, 1980:327-32.

5 Public Health Laboratory Service. Incidence of surgical wound infection in England and Wales. Lancet 1960;ii:659-63.

6 Postoperative wound infections. The influence of ultraviolet irradiation of the operating room and of various other factors. Ann Surg 1964;160 (suppl): $1-192$

(Accepted 3 December 1991)

\title{
Motor neurone disease: a hospice perspective
}

\author{
Tony O’Brien, Moira Kelly, Cicely Saunders
}

\begin{abstract}
Objective-To describe and evaluate the management of patients with motor neurone disease from the perspective of a hospice.
\end{abstract}

Design-Retrospective analysis of hospice medical and nursing notes.

Setting-Established 62 bed teaching and research hospice.

Subjects-124 patients with motor neurone disease cared for by the hospice between January 1980 and November 1990.

Main outcome measures-Patient profile; functional status; symptom control and use of opioids; insight; mode and management of death.

Results -124 patients (67 women, 57 men) had a mean age 63.9 years. The median length of admission was 61.5 days (range 1 to 2147). 84 patients $(68 \%)$ were aware of their diagnosis and its implications when first seen by a hospice doctor. Functionally, the patients were very dependent. Symptoms such as pain, dyspnoea, and insomnia were major problems that responded well to opioids. Many patients were noted to deteriorate "suddenly," and in $58 \%$ of cases death occurred within 24 hours of this deterioration. When dying, 106 patients (94\%) were peaceful and settled. 101 patients $(89 \%)$ received opioids during this dying period. No patient choked to death.

Conclusions-Although motor neurone disease is an uncommon disorder, many of its symptoms occur commonly in medical practice and must be actively treated. Opioids are both safe and effective for such treatment. The term choking is both inaccurate and inappropriate in describing the cause of death in motor neurone disease and its use should be abandoned.

\section{Introduction}

Motor neurone disease is a progressive disorder of unknown aetiology for which there is no cure. This sometimes equates with the belief that there is "nothing more to be done." This feeling of hopelessness and despair is frequently sensed by patients and families.

In hospice practice, the family is the unit of care and particular attention is paid to the prompt and effective relief of distressing symptoms in conjunction with an appropriate level of psychosocial and spiritual support. There is opportunity to explore the nature of the illness and to address specific fears and anxieties, which are an inevitable concomitant of any progressive, invariably fatal disease. The pace of such discussions is strictly dictated by the patient and family. Death is not always neat and tidy, but a peaceful death is more likely to be achieved when the patient has received an optimal level of physical and emotional care throughout the illness. Hospice is a concept of care that can be applied in any setting.

\section{Methods}

Application for admission to the hospice was considered at the request of the patient's general practi- 